\title{
Reintegro Laboral en Trabajadores con Problemas de Salud Mental: La Perspectiva de los Tratantes
}

\author{
RETURN TO WORK IN WORKERS WITH MENTAL HEALTH PROBLEMS: ATTENDANTS PERSPECTIVE
}

\author{
Alemka Tomicic', Claudio Martínez², Elisa Ansoleaga ${ }^{3}$, Pablo Garrido4, Claudia Lucero ${ }^{5}$, Soledad Castillo ${ }^{6}$, Constanza Dominguez ${ }^{7}$ \\ 1. Doctora en Psicología (PhD). Facultad de Psicología, Universidad Diego Portales, Chile. Iniciativa Científica Milenio Proyecto NS100018, Pontificia \\ Universidad Católica de Chile, Chile. \\ 2. Doctor en Psicología (PhD). Clínica Psicológica Universidad Diego Portales, Chile. Facultad de Psicología, Universidad Diego Portales, Chile. \\ 3. Doctora en Salud Pública (PhD). Programa de Estudios Psicosociales del Trabajo, Facultad de Psicología, Universidad Diego Portales, Chile. Clínica \\ Psicológica Universidad Diego Portales, Chile. \\ 4. Master of Sciences in Occupational Psychology $(\mathrm{MsC})$. Subgerencia de Salud Curativa, Asociación Chilena de Seguridad ACHS, Chile. \\ 5. Magister en Terapia Familiar. Clínica Psicológica Universidad Diego Portales, Chile. Pontificia Universidad Católica de Valparaíso, Chile. \\ 6. Psicóloga. Programa de Estudios Psicosociales del Trabajo, Facultad de Psicología, Universidad Diego Portales, Chile. \\ 7. Psicóloga. Programa de Estudios Psicosociales del Trabajo, Facultad de Psicología, Universidad Diego Portales, Chile.
}

\section{RESUMEN}

Antecedentes: En los últimos años se ha observado un aumento en la prevalencia de problemas mentales de origen laboral, incrementando significativamente el promedio anual de días de trabajo perdidos por reposo. Con respecto al posterior reintegro, la evidencia es categórica en afirmar la importancia de que este sea un proceso exitoso para la prevención de recaídas del trabajador. Asimismo, se constata que a nivel latinoamericano es casi inexistente la literatura e investigación en torno a intervenciones para el retorno laboral. Objetivo: Estudiar las condiciones de diagnóstico, intervención y retorno al trabajo que han mostrado efectividad desde la perspectiva y experiencias de tratantes de una mutualidad respecto de la intervención para el reintegro al trabajo en individuos con problemas de salud mental de origen laboral. Método: Se diseñó una investigación exploratoria-descriptiva, empleando metodología cualitativa. Se utilizaron grupos focales y entrevistas a seis psicólogos, tres psiquiatras y cuatro terapeutas ocupacionales que ejercen sus labores profesionales en una mutual en Chile. Resultados: A partir de un análisis descriptivo, empleando el procedimiento de codificación abierta propuesto por el modelo de Teoría Fundamentada, emergieron cinco categorias centrales y dos fenómenos transversales: La comunicación tripartita y el proceso orientado al reintegro laboral. Ambos hacen referencia tanto al proceso de intervención para el retorno al trabajo como a los actores involucrados en este, ya sea de la mutualidad como también del contexto laboral inmediato de los trabajadores.

(Tomicic A, Martínez C, Ansoleaga E, Garrido P, Lucero C, Castillo S, Domínguez C, 2014. Reintegro Laboral en Trabajadores con Problemas de Salud Mental: La Perspectiva de los Tratantes. Cienc Trab. Sep-Dic; 16 [51]: 137-145).

Palabras Claves: RETORNO AL TRABAJO, ESTRÉS LABORAL, METODOLOGÍAS CUALITATIVAS.

\section{ABSTRACT}

Background: In recent years there has been an increase in the prevalence of work-related mental problems, significantly increasing the average annual working days lost because of leave sick. With respect to the later return-to-work, the evidence is categorical in affirming the importance of the successful of this process for the relapse prevention. Furthermore, we note that throughout Latin America the literature and research on interventions to return-towork is almost absent.

Objective: To study the conditions of diagnosis, intervention and return to work that have shown effectiveness from the perspectives and experiences of professionals from a mutuality regarding the intervention to return to work in individuals with problems of mental health of occupational origin.

Method: An exploratory-descriptive research was designed using qualitative methodology. Focus groups and interviews to six psychologists, three psychiatrists and four occupational therapists were used.

Results: From a descriptive analysis using the procedure of open coding proposed by the Grounded Theory model, five core categories and two transverse phenomena emerged: The tripartite communication and the process oriented by the return to work. The two refer to the process of intervention to return to work as the actors involved in this, whether mutuality or the workers' immediate labor context.

Key words: RETURN TO WORK, WORK STRESS, QUALITATIVE METHODOLOGIES.
Correspondencia / Correspondence:

Alemka Tomicic

Grajales 1898. Santiago Centro. Santiago de Chile

e-mail: atomicic@gmail.com

Tel.: +56 977096048

Recibido: 26 de Agosto 2014 / Aceptado: 05 de 0ctubre 2014

\section{INTRODUCCION}

En los últimos años se ha observado un aumento sustantivo de la demanda de atención en salud mental para enfermedades profesionales, es decir, aquellos problemas de salud mental que son asociados al trabajo. Esto, desde la perspectiva de la salud pública, implica una multiplicidad de costos psicosociales y económicos que impactan a todos los actores involucrados. , $2,3,4,5,6^{-}$ 
El trabajo es una actividad central en la articulación de la vida cotidiana de las personas. La relevancia del trabajo como experiencia y práctica social radica, por un lado, en la gran dedicación temporal que se le asigna -se dedica al trabajo 50 de los 78 años de esperanza de vida al nacer ${ }^{7}-; y$, por el otro, en el conjunto de funciones psicosociales que cumple -como acceder a intercambios sociales, estructuración del tiempo, desarrollo del sentido de autoeficacia, y determinación de la autoestima e identidad personal-8,9,10,11. En las últimas décadas las transformaciones económicas, sociales y tecnológicas han afectado fuertemente la organización del trabajo, observándose procesos de intensificación, flexibilización, precarización de las condiciones laborales y, consecuentemente, una creciente inseguridad en el empleo. ${ }^{8,12,13}$ Estos procesos demandan y tensionan al trabajador/a emocional y cognitivamente, lo que se traduce en un aumento de la denominada carga psíquica y mental en el trabajo, la que reporta diferentes riesgos a la salud de las personas. ${ }^{14,8,9,15,16} \mathrm{En}$ los ambientes de trabajo, hoy por hoy conviven los clásicos riesgos físico-ambientales y los denominados riesgos psicosociales laborales. ${ }^{18,19,20}$

La visibilidad de los problemas de salud mental como un asunto de salud pública en América Latina y el mundo ha sido un proceso lento, empujado principalmente por cuatro cuestiones esenciales: los alarmantes indicadores de aumento de su prevalencia a nivel internacional; el impacto de este tipo de patologías en indicadores de discapacidad y mortalidad evitables; el reconocimiento de la comorbilidad entre estos y otros problemas sociales y de salud; y la alta carga económica asociada a la disposición de servicios de atención como también producto de la pérdida de productividad a nivel individual y de las organizaciones ${ }^{8}$. Chile no ha sido ajeno a esta realidad psicosocial. En el reporte de la primera Encuesta Nacional de Empleo, Trabajo, Salud y Calidad de Vida de los trabajadores y trabajadoras en Chile ${ }^{21}$, se señala que un 21\% de los trabajadores refiere haberse sentido melancólico, triste o deprimido por un período de dos semanas los últimos 12 meses, lo cual es significativamente mayor en las mujeres. ${ }^{22}$ Respecto de la prevalencia de patología mental en Chile y su asociación con incapacidad laboral se ha observado que las licencias médicas por trastornos mentales y del comportamiento de origen común experimentaron un alza de un 82\% entre los años 2005 y 2007. Las patologías asociadas a esta alza fueron depresión, ansiedad y estrés en el tramo de edad entre 20 y 40 años $^{23}$, convirtiéndose para el 2008 en la primera causa de incapacidad transitoria en los beneficiarios del Fondo Nacional de Salud. ${ }^{8,24}$ Algo similar ocurre en las Instituciones de Salud Previsional (ISAPRE), en las que las licencias por trastornos mentales aumentaron de un 15,3\% en el año 2005 a un 20,4\% en el año $2008 .^{25}$

La relación entre el trabajo y la salud mental ha cobrado particular importancia a partir de los efectos que los cambios en el trabajo han provocado en la salud de las personas. Según estudios de la Asociación Chilena de Seguridad ${ }^{26}$ las afecciones de salud mental de origen laboral aumentaron en un 87\% en los últimos 12 años; representaron un 24\% del total de las enfermedades profesionales acogidas en la ACHS en el 2012; acumularon 12.726 días perdidos por reposo laboral en el mismo período, que representan el 48\% del total de días perdidos por enfermedad profesional; y el 40\% de los costos curativos asociados a enfermedades profesionales correspondieron a enfermedades de salud mental ocupacional durante 2012. Por tanto, convierte a la salud mental vinculada al trabajo en un problema de salud emergente y representa un desafío para los actuales paradigmas de intervención en salud ocupacional y orientada al retorno laboral.
Por tanto, la magnitud de este problema nos sugiere que el estrés y la salud mental en el trabajo son enfermedades emergentes y representan un desafío para los actuales paradigmas de intervención en salud ocupacional y orientada al reintegro laboral.

El servicio de salud mental del Hospital del Trabajador de Santiago de Chile ha sido el primer servicio del sistema mutual en documentar la experiencia y sistematizar la atención de problemas de salud mental derivados del trabajo ${ }^{27,28}$, transfiriendo esta experiencia a las otras mutualidades del sistema. En este sentido, se ha avanzado en el desarrollo de criterios y protocolos para catalogar y sistematizar la calificación de la enfermedad de salud mental como laboral o no-laboral. Aun cuando este servicio ofrece tratamiento a trabajadores diagnosticados con problemas de salud mental calificados de origen laboral, es el impacto en días perdidos por reposo laboral y el alto número de días de tratamiento con reposo (53,12 días en promedio), lo que hace necesario revisar y proponer intervenciones más específicas donde el rol laboral es el factor etiológico principal. La evidencia es categórica en afirmar la importancia del reintegro exitoso al lugar del trabajo como un factor relevante para la prevención de recaídas del trabajador. ${ }^{14,29,3,20,30}$ Resultados de investigación a nivel internacional sobre retorno al trabajo identifican factores asociados al paciente ${ }^{31,32}$; factores asociados al tratamiento ${ }^{14,12}$; y factores asociados al entorno laboral. ${ }^{1,33}$ Ansoleaga et al. ${ }^{34}$ mencionan como facilitadores del retorno al trabajo -frecuentemente señalados por la literatura científica internacional para el caso de trabajadores con problemas de salud mental de origen laboral- los relacionados con características del trabajador (ej. percepción de autoeficacia, motivación hacia el trabajo), del ambiente de trabajo (ej. buena comunicación entre actores del proceso, el apoyo competente del supervisor; la evaluación de la capacidad del trabajador de manejar la carga de trabajo), y del tratamiento (ej. abordajes multidisciplinarios, con foco en reducción del estrés y entrenamiento de habilidades de afrontamiento específicas, una atención de salud oportuna junto a un plan de tratamiento).

En una revisión sistemática reciente ${ }^{35}$ se concluye que la terapia cognitivo-conductual y los tratamientos entregados en el lugar de trabajo, comandadas por terapeutas, psicólogos y psiquiatras, son las formas de intervención preferidas, y se señala como una figura nueva y relevante, pero poco considerada en los tratamientos habituales, el supervisor de retorno al trabajo. Asimismo se señala que de los 63 estudios revisados en esta investigación, el porcentaje mayor correspondió a los realizados en países europeos, destacando entre ellos Holanda. Por su parte el 80\% de los estudios abordaron el proceso de retorno laboral empleando preferentemente diseños de investigación de tipo cuantitativo, descriptivo y longitudinal, enfocados en indicadores para pronosticar factores protectores del tratamiento y de alerta para la relación terapia y reintegro al trabajo. Lo anterior muestra la casi inexistente literatura empírica a nivel latinoamericano, y la necesidad de producir conocimiento al respecto, tomando en consideración aproximaciones que permitan recoger e integrar las características contextuales de este fenómeno en este continente y en nuestro país.

En el presente estudio, como parte de una investigación mayor sobre los indicadores de efectividad en intervenciones para el reintegro al trabajo y evaluación de su impacto preventivo de recaídas $^{36}$, se aborda la experiencia de quienes han sido testigos de la creciente demanda en salud mental laboral y las maneras cómo la han afrontado en sus respectivos contextos. En este sentido, el propósito de esta investigación fue el de describir las 
condiciones de diagnóstico, intervención y retorno al trabajo que, a juicio de un grupo de tratantes de una mutualidad, han mostrado efectividad.

La generación de un conocimiento situado y contextualizado en la práctica y experiencia cotidiana de trabajadores del ámbito de la salud ocupacional acerca de factores involucrados en un retorno laboral efectivo y sustentable -y de estrategias en las que se han incorporado exitosamente variables que contribuyen a la disminución de recaídas y de prescripción de licencias médicas relacionadas con los problemas de salud mental tratados- contribuye a instalar esta línea de investigación en el ámbito nacional, posibilitando que sus resultados, considerando las condiciones socio-contextuales específicas, informen a la intervención en salud mental ocupacional de manera pertinente.

\section{MÉTODO}

El diseño del estudio fue exploratorio-descriptivo. Consecuentemente, empleando una metodología cualitativa, se indagó en las perspectivas y experiencias de tratantes de una mutualidad respecto del proceso de intervención para el reintegro al trabajo en el caso de individuos con problemas de salud mental de origen laboral.

\section{Participantes}

Los participantes correspondieron a seis psicólogos, tres psiquiatras y cuatro terapeutas ocupacionales que ejercen sus labores profesionales en una mutual en Chile y que habitualmente atienden pacientes diagnosticados con trastornos de salud mental calificados de origen laboral. El caso estuvo compuesto por seis mujeres y siete hombres, con un promedio de siete años de antigüedad en el cargo con un rango de uno y 21 años. Cuatro de los participantes trabajaban en la sede de la mutual correspondiente a la Región Metropolitana, en la ciudad de Santiago, capital de Chile, y nueve en sedes de otras regiones del país.

Los criterios de selección de los participantes siguieron las indicaciones de un muestreo de variedad máxima ${ }^{37}$ de modo que representaran la variedad de perspectivas en torno al objeto de estudio.

\section{Técnica de Recolección de Datos e Instrumento}

Para la recolección de datos se realizaron tres grupos focales (11 participantes en total) y cuatro entrevistas temáticas semiestructuradas (dos de los entrevistados participaron a su vez en los grupos focales). ${ }^{38}$ La decisión de combinar estas técnicas se fundamentó en un criterio práctico, vale decir, de acuerdo con las condiciones de posibilidad de acceso a los participantes. Se consideró que al tratarse de una recolección de tipo focal o temática, la producción de información en ambas instancias conversacionales incorporó integradamente tanto el discurso público como privado en torno al fenómeno estudiado. Por estas razones, en conjunto, los grupos focales y las entrevistas individuales fueron consideradas herramientas de producción de un corpus de análisis único.

Estas entrevistas fueron realizadas por los miembros del equipo de investigación. Para implementar los grupos focales y entrevistas se elaboraron guiones temáticos a partir de los objetivos especificos y preguntas directrices que orientaron el estudio, cuyas consignas invitaban a los entrevistados a responder desde su rol y experiencia como tratantes de problemas de salud mental de origen laboral y sobre tres grandes temáticas: (a) Evaluación e indicación: Orientada al proceso de evaluación de enfermedad profesional y los criterios de indicación de tratamiento; (b) Proceso terapéutico y resultados: Dirigida a caracterizar los objetivos y acciones de la intervención para el retorno al trabajo en el caso de problemas de salud mental de origen laboral; y (c) Resultados: Orientada a caracterizar los resultados esperados de la intervención así como los criterios para evaluarlos como exitosos.

\section{Procedimiento de Análisis}

Tanto a las transcripciones de los grupos focales como de las entrevistas se les aplicó un análisis cualitativo descriptivo. Específicamente, se implementó el procedimiento de codificación abierta, descrito en el método de la Teoría Fundada. ${ }^{39,38,40,41}$ El propósito de este análisis fue el de generar de manera inductiva conceptos y categorías respecto a la perspectiva de los tratantes sobre el proceso de intervención para el reintegro al trabajo en el caso de pacientes con problemas de salud mental de origen laboral, emergentes de las respuestas aportadas por los participantes. Es decir, caracterizar sus respuestas a través de un proceso basado en el discurso específico de los participantes y por medio de dos operaciones analíticas básicas: Formulación de preguntas (¿De qué "habla" este extracto en el marco de la pregunta de investigación?), y de hacer comparaciones (¿Este extracto "habla" de lo "mismo" que ya se ha conceptualizado en extractos anteriores?). Como resultado de este análisis se obtuvieron conceptos y categorias organizadas en esquemas de clasificación jerárquicos que se presentan en la sección de resultados.

Para asegurar la calidad de los resultados, fue empleada la estrategia de triangulación de interpretantes ${ }^{37}$, participando todos los investigadores en el proceso de análisis cualitativo de los datos, de manera que las categorías generadas fueron validadas a través de un acuerdo intersubjetivo. ${ }^{38}$ Asimismo, la generación de categorías y subcategorias fue orientada por el criterio de descripción dens $a^{42}$; es decir, procurando comprender e interpretar contextualizadamente las respuestas de los participantes de manera de describir plausiblemente los diversos aspectos implicados en el proceso de intervención para el retorno al trabajo en pacientes con problemas de salud mental de origen laboral. De esta manera, no se buscó obtener resultados generalizables, sino más bien generativos, en el sentido de describir la diversidad de expresión del fenómeno estudiado e inaugurar una línea de discusión e investigación.

\section{RESULTADOS}

Del análisis descriptivo de los grupos focales y entrevistas emergieron cinco categorías centrales:

- Etapa de calificación

- Actitud de las empresas

- Objetivos del tratamiento

- Reposo

- Reintegro

A continuación se expone la descripción de los conceptos y propiedades de cada una de estas categorías centrales, junto con sus respectivos esquemas de clasificación jerárquicos.

\section{Etapa de calificación}

La categoria Etapa de calificación (ver Figura 1) hace referencia a las menciones de los entrevistados de los aspectos implicados en el proceso de calificación de un problema de salud mental como de 
origen laboral (o enfermedad profesional, EP). En relación a lo anterior, como parte de este proceso son mencionadas la realización del estudio de personalidad, del estudio de puesto de trabajo (EPT) y, en algunos casos, la participación en este proceso de un psicólogo laboral. Ahora bien, asociado a las menciones del diagnóstico más general de una enfermedad profesional (EP), emergen imaginarios y representaciones acerca del paciente con un diagnóstico de neurosis laboral, que incluyen: el caso general del paciente de salud mental, el caso específico del paciente con depresión, el caso del paciente con múltiples 'recaídas', el caso del paciente que busca una ganancia secundaria y la vulnerabilidad del paciente.

Tal como se evidencia en la Tabla 1, los entrevistados señalan que si bien en ambos tipos de patología es posible observar dificultades frente al reintegro al trabajo, en relación a los problemas de salud mental, es más difícil, toda vez que las resistencias al retorno laboral son las mayores.

Tabla 1.

Extractos de opiniones vinculadas con la Etapa de Calificación.

\section{Identificación Texto}

FG1: 343 En el caso de pacientes de patología física, tienen resistencia también para volver, muchos por miedo, pero en el fondo del corazón hay algunos que quieren volver, o sea, no tenemos tanta reticencia como si la tenemos en salud mental.

FG3: 448 Es más fácil corroborar un estrés laboral. Un independiente, [independiente de cuál sea él] agente de riesgo, por así decir, es más fácil que en los lugares públicos. En las municipalidades, cuando la entrevista me la da quien (...) lleva como cuarenta años y dice la verdad de todo: Me da lo mismo si está tal alcalde o tal otro porque no me pueden despedir. Es distinto en la empresa privada, ahi es un poco más dificil que digan la verdad.

FG3: 40 Desarrollamos una dinámica de trabajo específicamente para tratar de acortar la latencia en la calificación. Porque, uno, encontramos que el paciente que era calificado con neurosis laboral retrasaba un mes 0 más su tratamiento porque habia que tener un compás de espera; dos, porque el paciente que no era calificado con neurosis laboral también retrasaba su tratamiento en volver a su previsión, a su trabajo y seguir; y tres, porque las cosas a la vista económica, el retraso en la calificación, también hacia que la parte administrativa de la agencia nos apurara un poco en calificar los casos.

Por su parte, otro de los entrevistados puntualiza sobre las dificultades para el proceso de calificación según el ámbito de trabajo y la posibilidad o no de un despido como consecuencia de la conclusión de que su problema de salud mental es de origen laboral, comparando la contratación en ámbitos públicos con el ámbito privado.

Finalmente, y en relación con la etapa de calificación, son mencionadas las condiciones de calidad de este proceso, señalando la importancia de una comunicación clara y oportuna tanto al paciente como al empleador, del cumplimiento y establecimiento de plazos apropiados. Con respecto a este último punto, en uno de los grupos focales se profundiza sobre una dinámica de trabajo que, como condición de calidad, ha logrado disminuir los tiempos y plazos en el proceso de calificación.

Las razones para acortar los tiempos en la calificación del trabajador con un problema de salud mental de origen laboral, dicen relación no solo con asegurar un tratamiento oportuno para el paciente acogido en la mutualidad, sino también para el caso del paciente no calificado que debe acudir al sistema común de atención en salud. Por otra parte, y como un tercer punto, las razones también tienen en consideración el contexto institucional y administrativo en el que, se podría hipotetizar, pudiese estar implicada la empresa.
Figura 1.

Esquema de Clasificación Jerárquico de la Categoria Etapa de Calificación.

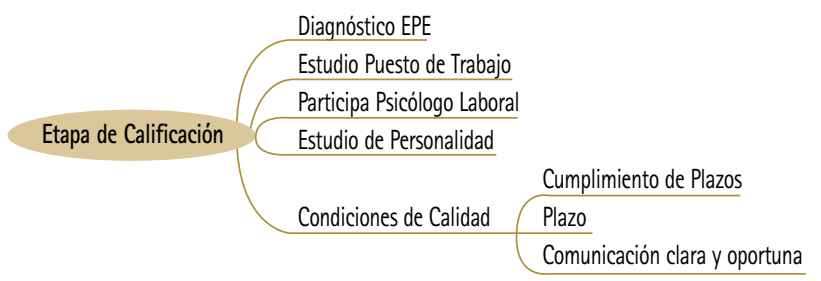

\section{Actitud de las empresas}

La categoría Actitud de las empresas (ver Figura 2) hace referencia a aquellos aspectos facilitadores y obstaculizadores mencionados por los entrevistados en miras al proceso de reintegro al trabajo (RT) de los trabajadores calificados con una enfermedad profesional (EP). Los facilitadores mencionados son menos en variedad que los obstaculizadores, y dicen relación con una buena comunicación entre la mutualidad y la empresa; la consideración de ambos -paciente y empresa- como beneficiarios de la mutualidad; la posibilidad de explicar y clarificar a la empresa el sentido y racionalidad del estudio de puesto de trabajo (EPT) y, finalmente, tener tacto en la relación con la empresa. Por su parte, los entrevistados mencionan que los obstaculizadores dependen del agente de riesgo (ligado a evento versus ligado a procesos organizacionales) y, específicamente, los identifican como relativos a la normativa (falta de normativa sobre el reintegro progresivo) y como relativos a la relación con la empresa (la atención de dos clientes que son contraparte -trabajador y empresa-, la extensión "injustificada" de la intervención, la falta de comunicación del EPT, no contar con la información de la licencia por enfermedad del trabajador, y tener que reconocer la responsabilidad por parte de la empresa, es decir, el origen laboral del problema de salud mental).

Tabla 2.

Extractos de opiniones vinculadas con la actitud de las empresas.

Identificación Texto

FG1: $75 \quad V a$ a depender de la justificación de la planificación porque varía mucho en los casos; o sea, cuando uno va por una hostilización la recepción de la empresa frente a esa justificación, son siempre malas, te podría decir que el $100 \%$ son malas; pero si es un evento, por ejemplo, que es una neurosis laboral por sobrecarga de trabajo, eso es mucho más fácil de comprobar... no sé, hizo 100 horas extras, efectivamente tuvimos un proceso de sobrecarga, de peak de producción, faltaron muchas personas con licencia médica, entonces en eso es mucho más fácil de aceptar.

E1: 33-35 Ahi nosotros no tenemos que calificar, o nosotros notificar al paciente, no tenemos que hacer ninguna de esas cosas, esa es la gran diferencia. Entonces se hace como más fácil, por asi decirlo, porque llega un estrés postraumático y nosotros lo atendemos (...); pero si llega una neurosis nosotros tenemos que evaluarlo, ingresarlo, estudiar el caso, notificar el caso, notificar a la empresa, todo ese proceso, entonces esa es la gran diferencia entre uno y otro.

Se menciona en uno de los grupos focales y una de las entrevistas cómo -ante un agente de riesgo ligado a un evento agudo, como sobrecarga de trabajo o estrés (ver Tabla 2)- resulta más fácil la calificación del problema como de origen laboral y la recepción de esta calificación por parte de la empresa, en comparación con el caso de un proceso de hostigamiento.

\section{Objetivos del tratamiento para el reintegro al trabajo}

La categoria Objetivos del tratamiento (ver Figura 3) hace referencia a las menciones de los psicólogos, psiquiatras y terapeutas 
Figura 2.

Esquema de Clasificación Jerárquico de la Categoria Actitud de las Empresas.

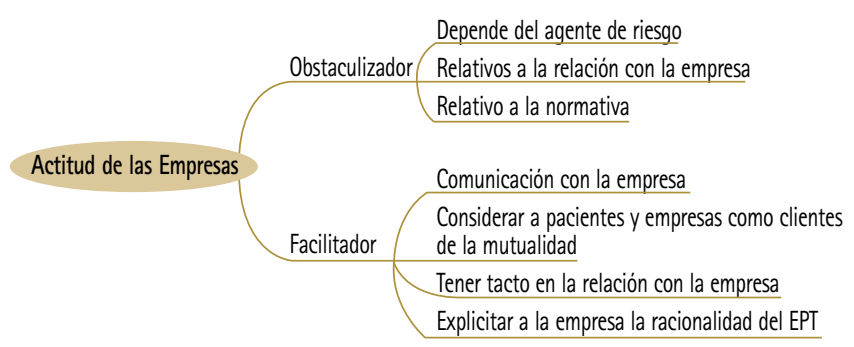

ocupacionales entrevistados sobre los objetivos específicos de su intervención en el caso del reintegro de pacientes calificados con neurosis laboral. Específicamente, tanto psicólogos como psiquiatras plantean cinco objetivos: El desarrollo de estrategias de autocuidado, la disminución de la sintomatología, contribuir a la elaboración de la experiencia por parte del paciente, fomentar la evaluación de formas de reintegro laboral y apoyar en el paciente la preparación para el reintegro al trabajo.

Por su parte, la categoría de Intervención del terapeuta ocupacional (T0) hace referencia, principalmente, a las menciones de los T0 de tres objetivos: La promoción en el paciente de un equilibrio ocupacional a través del desarrollo de la autonomía, la promoción en el paciente de un equilibrio ocupacional a través de su "productividad" y la promoción del retorno al trabajo. Este último objetivo, a la vez que es el aspecto central que articula todas las acciones terapéuticas, es recurrentemente mencionado por los diferentes actores como una meta final de toda intervención en este contexto (ver Tabla 3). En este sentido, señalan la importancia de trabajar en primera instancia a nivel de sintomatología, luego el desarrollo de habilidades para el trabajo y, en tercer lugar, sobre las condiciones para el reintegro laboral.

Adicionalmente, los entrevistados en conjunto hacen mención de las fronteras que suelen transitar los profesionales en su intervención. Las fronteras establecen los limites con los que se encuentran los tratantes y que señalan lo que puede llegar a ser conflictivo y lo que no en la relación entre tratante y trabajador y, entre el tratante y la mutualidad con la empresa. En otras palabras, constituyen las variaciones constantes entre conflicto y equilibrio entre lo que es "permitido" realizar en el tratamiento y lo que es "posible" de solicitar a la empresa para cambiar las condiciones que han enfermado al trabajador. Los entrevistados identifican tres tipos de fronteras: (a) relativas a la empresa (frontera entre empresas "perjudicadas" y el paciente; frontera entre la mutualidad y la empresa; y frontera entre la empresa y el reintegro del trabajador); (b) relativas al paciente (frontera entre el agente de riesgo y el paciente; y frontera entre entorno laboral y el paciente); y (c) relativas al tratamiento (frontera entre horizonte de controles y el reposo laboral; y frontera en la relación entre médico y paciente).

Las fronteras más señaladas son las relativas a la empresa que, desde la perspectiva de los entrevistados, son las más importantes para la generación de condiciones óptimas para el reintegro de los trabajadores.

\section{El lugar del reposo en el proceso de reintegro al trabajo}

La categoría Reposo (ver Figura 4) hace referencia a todas las menciones que los/as entrevistados/as hacen sobre el reposo laboral en el contexto de la intervención con trabajadores califi-
Tabla 3.

Extractos de opiniones vinculadas con los objetivos del tratamiento.

\begin{tabular}{|c|c|}
\hline Identificación & Texto \\
\hline GF2: 101 & $\begin{array}{l}\text { Siempre se trabaja, por lo menos yo, primero la sintomatología. } \\
\text { Porque si alguien está deprimido o está ansioso o desestructurado } \\
\text { no va a poder presentarse a ninguna entrevista de trabajo o no va } \\
\text { a poder asumir ninguna carga de trabajo de ningún tipo. Cuando la } \\
\text { sintomatología pasa, tú trabajas el autocuidado, o yo trabajo el } \\
\text { autocuidado, comenzar a desarrollar algunas herramientas que te } \\
\text { permitan mantenerte una vez que vuelvas a trabajar. Y después de } \\
\text { eso se ve en qué condiciones está la persona para trabajar o a su } \\
\text { empresa o a otra. }\end{array}$ \\
\hline GF1: 353 & $\begin{array}{l}\text { Yo diría que, en promedio, no sé, se me ocurren seis meses con los } \\
\text { trastornos adaptativos; ahora de reintegros laborales depende, pero } \\
\text { el tratamiento es con el reintegro laboral, o sea, no es después } \\
\text { viene el reintegro laboral; de hecho, eso es lo que es corregido al } \\
\text { paciente, el paciente tiene el discurso (...) este: yo tengo que estar } \\
\text { bien para volver a trabajar, debo estar cero kilometro, yo me tengo } \\
\text { que sentir bien; eso es un error. }\end{array}$ \\
\hline GF1: 90 & $\begin{array}{l}\text { Porque, ¿qué es de lo que más se queja la empresa? Es que no está } \\
\text { informada, eso es de lo que más se queja... frente también a que están } \\
\text { o no de acuerdo con la situación, pero principalmente que no están } \\
\text { informadas de lo que está pasando. }\end{array}$ \\
\hline E1: 267 & $\begin{array}{l}\text { Siempre tratamos de mantener contacto con prevención de riesgos, } \\
\text { porque ellos son la cara de la empresa, entonces cualquier duda les } \\
\text { van a consultar a ellos. Yo no quiero que los expertos digan } \\
\text { cualquier cosa respecto si es laboral el caso, cuando en realidad } \\
\text { está en estudio, porque eso significa un conflicto para la empresa y } \\
\text { por qué nadie me vino a ver (...). Porque cuando una neurosis } \\
\text { ingresa a la mutualidad o una enfermedad profesional cualquiera, } \\
\text { siempre es laboral, siempre ingresan como laboral hasta que uno } \\
\text { dice que no es laboral, o sea a nosotros nos aparece enfermedad } \\
\text { profesional en estudio, pero en el sistema de las empresas le } \\
\text { aparece como enfermedad profesional, entonces el experto o el } \\
\text { ejecutivo de la empresa tiene que conocer los procesos, según mi } \\
\text { percepción, para explicar y para que eso no se preste para cosa, } \\
\text { que no existan malos entendidos, para cuando uno vaya hacer el } \\
\text { EPT exista la disposición de la empresa. }\end{array}$ \\
\hline E4: 93-94 & $\begin{array}{l}\text { Es que ahi con la empresa, es una relación bien especial, es una rela- } \\
\text { ción como tripartita en el fondo, en que está la empresa, está el } \\
\text { trabajador y estamos nosotros; nosotros tenemos dos clientes en estos } \\
\text { casos y dos clientes que son contraparte entre si. Entonces siento } \\
\text { como la disyuntiva un poco, que estos dos, los dos elementos nos } \\
\text { perciben como aliándonos con el otro: el cliente me dice, pucha es que } \\
\text { ustedes, claro, la empresa paga las cotizaciones y ustedes nos presio- } \\
\text { nan, entonces, por eso, van a favor de ellos. Y cuando la empresa dice: } \\
\text { claro, ustedes acogen a todos los pacientes porque asi aumenta la } \\
\text { [ganancia] y los sueldos. Te fijas, porque también está ese otro, } \\
\text { entonces es bien dificil manejar eso de tal manera de que queden } \\
\text { todos conformes. }\end{array}$ \\
\hline
\end{tabular}

Figura 3.

Esquema de Clasificación Jerárquico de la Categoría Objetivos de Tratamiento.

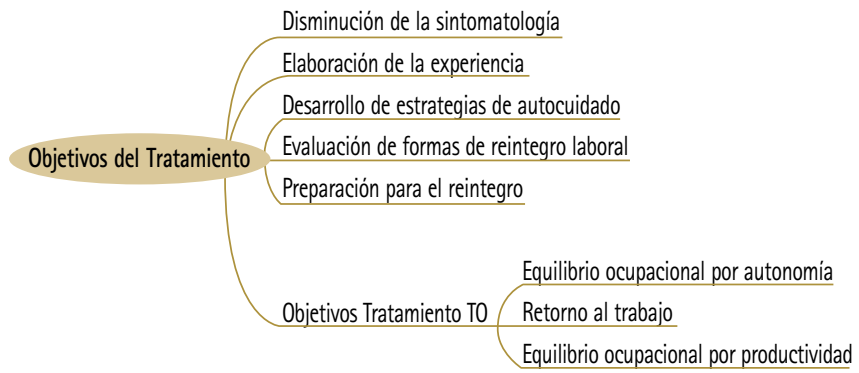


cados con problemas de salud mental de origen laboral en vistas al reintegro al trabajo. En primer lugar, se menciona que el reposo es una decisión exclusivamente médica y que tiene un fin orientado a manejar el estrés y la angustia en el paciente. Ahora bien, se señala también que el reposo es una medida habitual -que forma parte del protocolo actual de intervención- en aquellos trabajadores calificados con una enfermedad profesional (EP) y se lo piensa como un paréntesis de la vida cotidiana y una ayuda al tratamiento facilitando el reintegro al trabajo (promoviendo una mejor adaptación al entorno laboral). No obstante lo anterior, algunos de los entrevistados advierten que la extensión del reposo puede constituir un obstaculizador del reintegro laboral en el paciente, como queda expuesto en la Tabla 4.

\section{Figura 4.}

Esquema de Clasificación Jerárquico de la Categoría Reposo.

Reposo como medida habitual en pacientes calificados con EP
Reposo con fin estrictamente médico
El reposo como un paréntesis de la vida cotidiana
Reposos para enejar el estrés y la angustia
Facilita el retorno al trabajo (mejor adaptación)
Reposos como una desición exclusiva del psiquiatra
La extensión del reposo como obstaculizador del retorno al trabajo

Tabla 4.

Extractos de opiniones vinculadas al reposo en el proceso de reintegro.

\begin{tabular}{|c|c|}
\hline Identificación & Texto \\
\hline $\mathrm{E} 2: 46$ & $\begin{array}{l}\text { Tanto tiempo con reposo, a veces, termina siendo contraproducente, } \\
\text { porque, insisto, es una situación en que no se define como un } \\
\text { reposo que tiene fecha y tampoco son vacaciones. }\end{array}$ \\
\hline GF2:121-122 & $\begin{array}{l}\text { El tema del reposo es una ayuda al tratamiento, no es el } \\
\text { tratamiento en sí. El eje del tratamiento muchas veces lo constituyen } \\
\text { los fármacos y muchas veces la psicoterapia, el tema del reposo es } \\
\text { un elemento paralelo que ayuda. En ninguna ocasión se te ha } \\
\text { mencionado que el reposo o la licencia es el tratamiento. Siempre } \\
\text { vamos a tener que tener presente el tema de la iatrogenización del } \\
\text { paciente que, teniendo sus habilidades, si prolongamos en exceso } \\
\text { el reposo, va a asumir la condición de estar incapacitado o de estar } \\
\text { inhabilitado para ejercer alguna función: No, ya no soy capaz. Y } \\
\text { luego ya no se le pasa. Ahora es un tremendo tema. }\end{array}$ \\
\hline
\end{tabular}

\section{El reintegro al trabajo como resultado de la intervención}

La categoria Reintegro (ver Figura 5) hace referencia a las menciones de los entrevistados relativas al proceso y resultados del reintegro al trabajo en pacientes calificados con problemas de salud mental de origen laboral. Entre los aspectos señalados que se plantea el trabajo terapéutico dirigido al reintegro se incluyen: la generación de acuerdos sobre el retorno al trabajo con el paciente; el trabajo de desensibilización sistemática y la implementación de técnicas específicas; la exploración de características personales del paciente que pueden contribuir o no al retorno al trabajo; la relación del paciente con la empresa; y el tratamiento más allá del reintegro. Otro aspecto importante mencionado dice relación con la temporalidad del reintegro laboral. Se plantea de preferencia la realización de un retorno al trabajo de carácter progresivo, valorando que sea precoz, evitando así, entre otras consecuencias el prolongar el reintegro y la contaminación de los pacientes con informaciones informales de compañeros de trabajo, $\mathrm{u}$ otras personas vinculadas al contexto laboral, por ejemplo, abogados laborales.
Tabla 5.

Extractos de opiniones vinculadas al reintegro al trabajo.

\section{Identificación Texto}

GF2:225 (...) cuando el paciente está mucho tiempo con reposo, ahi se recomienda el reintegro progresivo (...). Entonces tratamos de que sea así.

E3:134 Desde que ingresan hasta que les toque el alta, que no necesariamente responde al momento en que empiezan a trabajar, porque muchas veces ellos se incorporan a trabajar entremedio y yo los sigo viendo igual.

GF1:158-165 M2: es que va a depender del caso. Porque si, por ejemplo, la persona que lo autorizaba ya no está en la empresa o ya no está en el puesto de trabajo, no se justifica hacer un reintegro progresivo porque el actor estresor ya no está. Y se seguiria extendiendo la licencia porque un reintegro progresivo de acuerdo.

H1: Una licencia parcial.

M1: No, completa el reintegro progresivo; es a modo de terapia. El paciente está con reposo y nosotros le seguimos pagando.

H2: Se siguen cargando los días perdidos a la empresa.

M1: La empresa sigue con los dias perdidos y eso si es un sistema que depende de la buena voluntad de la empresa porque la empresa no está, no es legal.

M2: No podemos obligar a una empresa a recibir a un paciente estando con licencia médica. Ellos pueden decir: ¿sabes? que nosotros rechazamos este sistema.

H1: Es un acto terapéutico entonces.

$\mathrm{M} 1$ : Si, exacto y tampoco puedes [sic], o sea, este paciente que era como un león enjaulado no puede ir a reintegro aunque el psiquiatra me lo pida, no, aunque la empresa me diga que sí, ino! Porque la idea es un preámbulo al alta, o sea, en cualquier momento dado, él podria haber sido dado de alta, pero creemos que terapéuticamente se va a beneficiar con este reintegro.

E6:113-116 Cuando hay un tema de hostigamiento u hostigamiento perseguido, claramente no va a haber confianza. Ahora hay otra tema, que vamos pasando al tema específico de ustedes, que esto de la comunicación mal hecha también influye mucho en la reinserción, porque está uno de nuestros motivos serios para reinsertar a la gente es que, sobre todo en casos complejos, es que el paciente se resista, no quiere volver (...). Porque tiene temor, tiene desconfianza, no sabe a lo que se va, salió mal de su empresa y han pasado dos meses y no ha tenido ningún contacto, solo el contacto que tiene son los rumores de los compañeros que los llaman de vez en cuando y le dicen, oye supiste que andan diciendo que aqui te van a despedir [sic], que esto otro, que... Asi son las comunicaciones, entonces eso genera una expectativa súper negativa y súper incierta del paciente y es muy dificil para nosotros poder revertir eso, también el cómo lo convencemos de que no es asi o que, a lo mejor no es así.

Figura 5.

Esquema de Clasificación Jerárquico de la Categoria Reintegro.

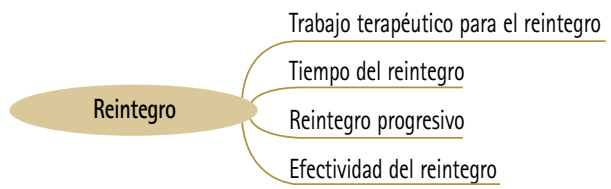

Respecto al reintegro progresivo, en uno de los grupos focales y en una de las entrevistas se lo señala como una alternativa cuando el tiempo de reposo ha sido muy extendido o para que no se extienda innecesariamente (ver Tabla 5).

Por su parte, y en relación a la "contaminación" de los pacientes, uno de los entrevistados lo asocia a los problemas en la comunicación de la situación de reposo por parte del trabajador y la proliferación bilateral de rumores que terminan dificultando el reintegro al trabajo.

Finalmente, se plantean algunos aspectos relativos a la efectividad del reintegro: La desconfianza, las resistencias (e. g. actitud del empleador, la personalidad del paciente y la relación empleadorempleado), y las recaídas que podrian afectar y ser indicadores 
negativos de la efectividad del reintegro. Por otro lado, a juicio de los entrevistados, intervención ambiental (empresa) y el seguimiento podrían incidir y ser indicadores positivos de la efectividad del retorno laboral.

\section{DISCUSIÓN}

Este estudio se realizó teniendo en cuenta la magnitud del problema de la salud mental ocupacional, específicamente como un ámbito emergente y que representa un desafío para los actuales paradigmas de intervención en salud ocupacional y de orientación al efectivo retorno al trabajo. El propósito de esta investigación fue levantar información sobre los factores asociados con estos objetivos, considerando la experiencia y perspectiva de uno de los actores involucrados: psicólogos, psiquiatras y terapeutas ocupacionales.

A partir de las cinco categorías emergentes -etapa de calificación, actitud de las empresas, objetivos de la intervención, reposo y retorno al trabajo- fue posible visualizar dos fenómenos transversales: Comunicación Tripartita y Proceso Orientado al Reintegro Laboral. Ambos hacen referencia tanto al proceso de intervención (desde el diagnóstico hasta el reintegro), como a los actores involucrados en este, ya sea de la mutualidad (psicólogos, psiquiatras y terapeutas ocupacionales) como también del contexto laboral inmediato (empleadores y compañeros de trabajo).

El primer fenómeno transversal dice relación con la comunicación tripartita entre la mutualidad, el empleado y el empleador. Así, a lo largo del proceso, desde la calificación al reintegro, destaca la importancia de una comunicación oportuna y fluida desde la mutualidad hacia el trabajador y hacia el empleador. No sólo parece relevante la forma cómo se realiza esta comunicación, sino también los diferentes contenidos que adquiere a lo largo del proceso. Por una parte, en la etapa de calificación resulta fundamental que la mutualidad comunique a la empresa en qué estado de la evaluación se encuentra el trabajador y sobre la extensión del reposo otorgado a este. Por otra, durante la etapa de intervención aparece como relevante la comunicación al paciente del sentido y extensión de la licencia, y generar una instancia de comunicación entre empleado y empleador para establecer las condiciones apropiadas para el reintegro laboral. Al respecto, estudios internacionales han destacado el papel de la comunicación entre los distintos actores involucrados ${ }^{43,44}$, y las características del diálogo entre el supervisor directo y el trabajador en los sentimientos y cogniciones del trabajador respecto a su lugar de trabajo, y su consiguiente reintegro. ${ }^{4,45}$ Karlson et al. ${ }^{45}$ relevan la importancia de que este diálogo se oriente hacia la búsqueda de un ajuste entre las expectativas del trabajador y las del supervisor respecto al desempeño del primero durante el reintegro, siendo fundamental una actitud del supervisor directo caracterizada por la apertura, flexibilidad, atención, empatía y apoyo. En relación con el momento de establecer esta comunicación tripartita, y en coherencia con lo planteado por los entrevistados en el presente estudio, los hallazgos respecto a la importancia del contacto precoz entre trabajador y empleador no son consistentes. Por un lado, algunas investigaciones han mostrado que el contacto temprano entre el empleador y el trabajador no siempre constituye un facilitador para el proceso de reintegro. ${ }^{30}$ Por otro, se enfatiza que mantener desde el inicio una actitud de apoyo del empleador hacia el trabajador permite preservar la relación laboral y promover su reintegro. ${ }^{43,46}$
El segundo fenómeno transversal dice relación con la comprensión del reintegro al trabajo como el foco que guía todo el proceso de atención de la mutualidad sobre un trabajador que es admitido por un problema de salud mental. Respecto de este fenómeno, emerge como un aspecto relevante, y que oficia de contexto, la idea de que la mutualidad ejerce un rol de mediación entre ambos beneficiarios: el trabajador y la empresa. En esta mediación, la mutualidad instalaría el reintegro al trabajo como el problema a resolver entre ambas partes. En este marco, surgen imaginarios y representaciones sobre las neurosis laborales que imprimen una particular concepción respecto a las dificultades que presenta la intervención y negociación del retorno al trabajo de los empleados. Así, en el caso de los problemas de salud mental -a juicio de los entrevistados- la intervención en vistas a un reintegro sería más difícil tanto por las características del trabajador (e. g. paciente difícil) como por las reticencias de la empresa a asumir la responsabilidad del problema (e. g. agente de riesgos vinculados a un proceso, por ejemplo, hostigamiento). No obstante estas dificultades, todos los entrevistados señalan que las intervenciones, tanto sobre el trabajador como sobre la empresa, debieran orientarse al retorno al trabajo. Esto ya ha sido señalado por Karlson et al. $^{46}$, quienes subrayan la necesidad de que la intervención tenga una clara orientación al lugar de trabajo, y que exista la posibilidad real de desarrollar cambios en la situación laboral, permitiendo un manejo beneficioso del proceso de rehabilitación. Asimismo, se ha observado que una atención psiquiátrica especializada en el ámbito ocupacional disminuye el tiempo de retorno al compararse con la atención habitual. ${ }^{5}$ En el proceso de tratamiento, se plantea como un ideal que el reintegro sea precoz y de carácter progresivo, que no sólo considere el trabajo de preparación sobre el trabajador, sino también sobre el contexto laboral que lo recibe, al modo de un nuevo EPT orientado al retorno efectivo. En otras palabras, el diseño de un estudio del puesto para el retorno al trabajo. Asociado con lo anterior, vale decir con las características específicas que debiese considerar una intervención orientada al trabajo, Muijzer et al. ${ }^{43}$ han señalado que esta debiese ser temprana y enfocada en el trabajador, en la reducción del estrés, el entrenamiento en habilidades, y en el apoyo del supervisor en el trabajo.

Por último, el reposo, como una categoría emergente, concita por sí misma un particular interés en los entrevistados y para el proceso de reintegro. La utilización de este instrumento (i. e. licencia médica) muchas veces resultaría desarticulada de otras intervenciones orientadas a este proceso de retorno laboral. El reposo se utilizaría indiscriminadamente, más por un estándar protocolar y/o legal que como un uso terapéutico, tal como fue su concepción de origen. Al mismo tiempo, al ser de uso y manejo exclusivo del médico-psiquiatra, queda a veces totalmente separado de la visión de los otros miembros del equipo, quienes podrían tener una postura diferente con respecto a este tiempo de reposo para el trabajador. Además, la experiencia de algunos equipos de trabajo entrevistados es que el reposo puede ser iatrogénico de la posibilidad de recuperación y reintegro laboral efectivo. Al respecto, Flach, Groothoff, Krol \& Bültmann ${ }^{33}$, en su investigación sobre primer retorno al trabajo y duración del reposo en trabajadores con problemas de salud mental observaron que, en el caso de licencias prolongadas, más que los síntomas es la predominancia de factores psicosociales que rodean al trabajador la que influye en el reintegro. No obstante 
lo anterior, y en un sentido aparentemente opuesto, estos autores constataron también que el diagnóstico clínico tiene una fuerte asociación con el proceso de reintegro y la duración total del reposo por enfermedad.

En conjunto, estos dos fenómenos emergentes y transversales comunicación tripartita, y el reintegro como foco y guía del proceso de intervención-, sugieren acciones específicas (por ejemplo, la consideración de los tiempos para el establecimiento de la comunicación entre los actores; la rapidez de la intervención y la orientación precoz de esta hacia el proceso de retorno; el uso de la licencia como una herramienta de intervención; el estudio del puesto de trabajo para generar condiciones óptimas para el retorno del trabajador) que se alinean con la literatura internacional que plantea una serie de prácticas para el manejo de la ausencia laboral y el retorno al trabajo. En una revisión sistemática reciente, estas prácticas son integradas en un modelo procesual y secuencial de seis etapas que consideran el período de reposo, el contacto precoz con el trabajador, una intervención que incorpore la evaluación del trabajador en su lugar de trabajo, el desarrollo de un plan de reintegro, el reintegro propiamente tal y, por último, un seguimiento del proceso de retorno al trabajo. ${ }^{47}$

\section{Fortalezas y limitaciones de la investigación}

Una limitación de este estudio es que no participan en él dos de los principales actores involucrados en el proceso de retorno al trabajo: trabajador y empresa. En este sentido, una comprensión más contextualizada sobre el fenómeno estudiado requeriría incorporar la perspectiva de los trabajadores y sus empleadores sobre este proceso. Sin embargo, los resultados obtenidos podrían constituir una buena base para estudios posteriores que triangulen, incorporando la visión de estos otros actores. Aun así, los hallazgos presentados, basados en las experiencias y conocimiento local, permiten esbozar guias de intervención que contribuyan a enriquecer los tratamientos e intervenciones actuales orientadas a un retorno al trabajo efectivo.

\section{Financiamiento}

Este trabajo recibió financiamiento de la Fundación Científica y Tecnológica de la Asociación Chilena de Seguridad (Proyecto FUCYT P0126/2012) y contó con el apoyo de la Iniciativa Científica Milenio del Ministerio de Economía, Fomento y Turismo, Proyecto NS100018.

\section{REFERENCIAS}

1. Lemieux, P. Durand, M. \& Hong, Q. Supervisors' perception of the factors influencing the return to work of workers with common mental disorders. Journal of Occupational Rehabilitation. 2011; 21(3):293-303.

2. Macdonald, S. Maxwell, M. Wilson, P. Smith, M. Whittaker, W. Sutton, M. \& Morrison, J. A powerful intervention: general practitioners'; use of sickness certification in depression. BMC family practice. 2012; 13: 82-91.

3. Lagerveld, S. E. Blonk, R. W. Brenninkmeijer, V. Wijngaards-de Meij, L. \& Schaufeli, W. B. Work-focused treatment of common mental disorders and return to work: a comparative outcome study. Journal of occupational health psychology. 2012; 17(2): 220-234.

4. Hees, H., Nieuwenhuijsen, K., Koeter, M., Bultmann, U. \& Schene, A.H. (2012). Towards a new definition of return-to-work outcomes in common mental disorders from a multi-stakeholder perspective. PLoS One. 2012; 7(6): 1-6

5. Van der Feltz-Cornelis, C. Hoedeman, R. Jong, F. Meeuwissen, J. Drewes, H. Van der Laan, N. \& Ader, H. Faster return to work after psychiatric consultation for sick listed employees with common mental disorders compared to care as usual. A randomized clinical trial. Neuropsychiatric disease and treatment. 2010; 6: 375-385.
6. D'Amato, A., \& Zijlstra, F. Toward a climate for work resumption: the nonmedical determinants of return to work. Journal of occupational and environmental medicine. 2010; 52: 67-80.

7. Organización Internacional del Trabajo. Evolución demográfica y pensiones en Chile. Seguridad social y reforma del sistema de pensiones en Chile. OIT Notas. Santiago. Chile. 2006

8. Ansoleaga, E. Sintomatología depresiva y consumo riesgoso de alcohol en trabajadores expuestos a factores psicosociales laborales adversos: Un asunto pendiente en Salud Pública. (Tesis doctoral inédita). Universidad de Chile, Facultad de Medicina, Escuela de Salud Pública, Chile. 2013

9. Dejours, C. Trabajo y desgaste mental. Una contribución a la psicopatología del trabajo. Editorial Humanitas. Buenos Aires. Argentina. 1992

10. Hees, H. de Vries, G. Koeter, M. \& Schene, A. Adjuvant occupational therapy improves long-term depression recovery and return-to-work in good health in sick-listed employees with major depression: results of a randomised controlled trial. Occupational and Environmental Medicine. 2012: 1-9.

11. Verdonk, P., de Rijk, A., Klinge, I., \& de Vries, A. Sickness absence as an interactive process: Gendered experiences of young, highly educated women with mental health problems. Patient Education and Counseling. 2008; 73(2): 300-306. 
12. Roelen, C., Norder, G. Koopmans, P. van Rhenen, W. van der Klink, J. \& Bültmann, U. Employees sick-listed with mental disorders: who returns to work and when?. Journal of occupational rehabilitation. 2012; 22(3): 409-417.

13. St-Arnaud, L., Pelletier, M., \& Briand, C. The Paradoxes of Managing Employees' Absences for Mental Health Reasons and Practices to Support Their Return to Work. Journal Social Action in Counseling \& Psychology. 2011; 3(2): 36-52.

14. Ahlstrom, L. Hagberg, M. \& Dellve, L. Workplace Rehabilitation and Supportive Conditions at Work: A Prospective Study. Journal of occupational rehabilitation. 2012; 23(2): 248-260.

15. Díaz, C. Actividad Laboral y Carga Mental de Trabajo. Ciencia \& Trabajo. 2010; 12(36): 281-292.

16. Tennant, C. Work-related stress and depressive disorders. Journal of Psychosomatic Research. 2001; 51(5): 697-704.

17. Willert, M., Thulstrup, A., \& Bonde, J. Effects of a stress management intervention on absenteeism and return to work-results from a randomized wait-list controlled trial. Scandinavian Journal Work, Environment \& Health. 2012; 37(3): 186-195.

18. Houtman, I., \& Kompier, M. Trabajo y Salud Mental. En J. Mager (Ed.), Enciclopedia de Salud y Seguridad en el Trabajo. 2001: 5.1-5.4. Disponible en http://www.insht.es/portal/site/Insht/menuitem.1f1a3bc79ab34c578c2e88840 60961 ca/?vgnextoid=a981 ceffc39a5110VgnVCM100000dc0ca8c0RCRD\&vgne xtchannel=9f164a7f8a651110VgnVCM100000dc0ca8c0RCRD

19. Instituto Sindical de Trabajo, Ambiente y Salud. Organización del Trabajo, Salud y Riesgos Psicosociales. Guía del delegado y delegada de prevención. Paralelo Edición. Barcelona. España. 2005. Disponible en http://www.istas.ccoo.es/ descargas/guia\%20de\%20sensibilizacion\%20def.pdf

20. Wang, J., Schmitz, N., Dewa, C., \& Stansfeld, S. Changes in perceived job strain and the risk of major depression: results from a population-based longitudinal study. American Journal of Epidemiology. 2009; 169(9): 1085-1091.

21. Chile. Ministerio de Salud, Ministerio del Trabajo \& Instituto de Seguridad Laboral. Informe Interinstitucional: Primera encuesta nacional de empleo, trabajo, salud y calidad de vida de los trabajadores y trabajadoras de Chile. 2011. Disponible en http://www.eligevivirsano.cl/wp-content/uploads/2012/01/ Informe-Encuesta-ENETS-2009-2010.pdf

22. Ansoleaga, E., \& Miranda, G. Depresión y condiciones de trabajo: revisión actualizada de la investigación psicosocial. 2011. Revista Costarricense de Psicología. 2014; 33 (1): 1-14.

23. Superintendencia de Seguridad. Licencias tramitadas por problemas de salud mental. Santiago. Chile. 2008

24. Fondo Nacional de Salud. Boletín Estadistico 2006-2007. Santiago. Chile. 2008.

25. Pezoa, M. Análisis de las licencias médicas curativas en Isapres 2007-2008. Superintendencia de Seguridad Social (SUSESO). Santiago. Chile. 2008.

26. Asociación Chilena de Seguridad. Salud Mental Ocupacional. Santiago. Chile. 2013. Recuperado de http://www.achs.cl/portal/Empresas/Paginas/Salud-Mental.aspx

27. Trucco, M., \& Rebolledo, P. Neurosis profesional o enfermedad común. Sintomas y estresores. Revista Médica de Chile. 2011; 139: 1370-1377.

28. Rebolledo, P. Neurosis Ocupacionales: Experiencia ACHS. Manuscrito no publicado. 2007

29. De Vente, W. Kamphuis, J. Emmelkamp, P., \& Blonk, R. Individual and group cognitive-behavioral treatment for work-related stress complaints and sickness absence: a randomized controlled trial. Journal of occupational health psychology. 2008; 13(3): 214-231.

30. Tjulin, A. Maceachen, E. \& Ekberg, K. Exploring the meaning of early contact in return-to-work from workplace actors' perspective. Disability \& Rehabilitation. 2008; 33(2): 137-145.
31. Ruwaard, J. Lange, A. Schrieken, B. Dolan, C. V., \& Emmelkamp, P. The Effectiveness of Online Cognitive Behavioral Treatment in Routine Clinical Practice. Plos ONE. 2012; 7(7): 1-9.

32. Nielsen, M. B. Madsen, I. E. Bultmann, U., Christensen, U. Diderichsen, F. \& Rugulies, R. Predictors of return to work in employees sick-listed with mental health problems: findings from a longitudinal study. European Journal Public Health. 2011; 21 (6): 806-811.

33. Flach, P. Groothoff, J. Krol, B. \& Bultmann, U. Factors associated with first return to work and sick leave durations in workers with common mental disorders. European Journal Public Health. 2012; 22(3): 440-445.

34. Ansoleaga, E. Garrido, P. Domínguez, C. Castillo, S. Lucero, C. Tomicic, A., \& Martínez, C. Facilitadores del Reintegro Laboral en Trabajadores con Patología Mental de Origen Laboral: Una Revisión Sistemática. en Prensa. Revista Médica de Chile 2015.

35. Garrido, P. Ansoleaga, E. Tomicic, A. Domínguez, C. Castillo, S. Lucero, C. \& Martínez, C. Afecciones de Salud Mental y el Proceso de Retorno al Trabajo: una Revisión Sistemática. Ciencia \& Trabajo. 2013; 15 (48): 105-113. En prensa.

36. Ansoleaga, E. Martinez, C. Garrido, P. Tomicic, A. \& Lucero, C. Indicadores de Efectividad en Intervenciones para el Reintegro al Trabajo y Evaluación de su Impacto Preventivo de Recaidas en Trastornos Depresivos Calificados de Origen Laboral (PROYECTO FUCYT P0126). Manuscrito no publicado. 2012

37. Patton, M. Q. Qualitative evaluation and research methods (2nd ed.). Sage Editorial. Newbury Park. California. 1990.

38. Flick, U. Introducción a la investigación cualitativa. Editorial Paideia. Madrid. 2004.

39. Charmaz, K. Constructing Grounded Theory. A practical guide through qualitative analysis. Sage. London. 2006.

40. McLeod, J. Qualitative research in counseling and psychotherapy. London: Sage. London. 2001.

41. Corbin, J. \& Strauss, A. Basics of qualitative research: Techniques and Procedures for Developing Grounded Theory (3rd ed.). Sage. London. 2008.

42. Ponterotto, J. G. (2006). Brief note on the origins, evolution, and meaning of the qualitative research concept "thick description". The Qualitative Report. 2006; 11: 538-349. Disponible en http://www.nova.edu/ssss/QR/ QR11-3/ponterotto.pdf

43. Muijzer, A. Brouwer, S. Geertzen, J.H. \& Groothoff, J.W. Exploring factors relevant in the assessment of the return-to-work process of employees on long-term sickness absence due to a depressive disorder: a focus group study. BMC Public Health. 2012; 12: 103-110.

44. Martin, M.H. Nielsen, M.B. Petersen, S.M. Jakobsen, L.M. \& Rugulies, R. Implementation of a coordinated and tailored return-to-work intervention for employees with mental health problems. Journal of occupational rehabilitation. 2012; 22(3): 427-436.

45. Karlson, B., Jönsson, P., Pålsson, B., Åbjörnsson, G., Malmberg, B., Larsson, B. \& Österberg, K. Return to work after a workplace-oriented intervention for patients on sick-leave for burnout -a prospective controlled study. BMC Public Health. 2010; 10: 301-310.

46. Muijzer, A. Groothoff, J.W. Geertzen, J.H. \& Brouwer, S. Influence of Efforts of Employer and Employee. Journal Occup Rehabil. 2011; 21(4): 513-519.

47. Durand, M. J. Corbière, M. Coutu, M. F. Reinharz, D. \& Albert, V. A review of best work absence management and return-to-work practices for workers with musculoskeletal or common mental disorders. Work Journal Prevention, Assessment and Rehabilitation. 2014; 48(4): 579-589. 